Session 2480

\title{
Illuminating Engineering
}

\author{
Laura J. Bottomley and Elizabeth A. Parry \\ North Carolina State University/Science Surround
}

\begin{abstract}
Engineering is a difficult profession to explain to the average person, much less student, and is probably one of the most frequently misunderstood. The session described in this paper was developed to put engineering in common terms for the lay person, as well as provide an interesting and fun way to explore different concentration areas of the profession. The demonstration has been given to children as young as six years old, to parents of incoming engineering freshman and to emeritus engineers for the purpose of highlighting how the profession has changed. Little adaptation is needed, surprisingly enough, for these diverse audiences.
\end{abstract}

Many of the demonstrations involve basic science as a way of illustrating the application of science to the solution of engineering problems. The session is heavily dependent on audience participation, making use of active learning. A sense of humor is also a necessary component of the presentation; it seems to help the audience become receptive to the ideas that are being presented.

For the purposes of the demonstration the working definition of engineering is as a creative profession that uses math and science as tools to solve problems. The wide range of potential work areas is hinted at, as is the essential integrated nature of the various engineering disciplines.

\section{Introduction}

What does an engineer do? To most people, even professionals who have worked in the field for years, this question is a challenge to answer. Simply put, engineers use math and science to solve problems. Even the kindergartner can understand this succinct definition. But what does it really mean? As part of its outreach program, the College of Engineering at North Carolina State University takes engineering to the K-12 campus to demonstrate the kind of work engineers perform. The session is appropriate for all ages, requiring only that the depth of the explanations be tailored to age appropriateness. The intent of the program is to inform, versus educate, about the engineering profession. The tone is light and a sense of humor is mandatory to help dispel preconceived notions about the field. Indeed, the tone of this paper is written in the manner best suited for the presentation itself, in a straightforward, approachable way.

Proceedings of the 2001 American Society for Engineering Education Annual Conference \& Exposition, Copyright 2001, American Society for Engineering Education 
The session begins with questions. What does an engineer do? What does a scientist do? Surprisingly enough, nonspecific answers are the common response among all age levels. Most of the children will know that scientists do experiments. Building on that knowledge, we agree that scientists use experimentation to discover new things. Engineers use the work developed by scientists to develop useful applications. Engineers may experiment too, and scientists develop useful things! The discussion naturally flows then into the kinds of subjects the students need to be proficient in to be an engineer. Most, if they were listening, will correctly guess that engineers have to be proficient in math and science. To elucidate the engineering definition we have arrived at, we then proceed with a series of short demonstrations to show the kind of work a particular engineering discipline might engage in.

\section{Demonstration}

Between 7-10 engineering disciplines can be demonstrated in a 50-60 minute session. In all cases, audience volunteers participate, led by the instructor. A brief explanation of the discipline is given, followed by the demonstration itself. It is important to remember that the point of the session is to inform and intrigue, not to educate about engineering principles. To that end, each demonstration is presented as but a tiny part of what an engineer of that particular discipline might do. Certainly, the audience understands the actual profession is much more involved.

\section{Aerospace and Aeronautical Engineering}

The equipment for this demonstration is simple: a toy "helicopter" (propeller on a twist stick) and some plain paper. Most children will quickly ascertain that this kind of engineer works on airplanes or the space program. This is a natural lead-in to a discussion about the many different things that "fly", including rockets, the space shuttle, satellites, and aircraft. We next call up an audience volunteer and ask them to demonstrate a hand-held spin copter, similar to the type the Wright brothers manufactured in their shop as they pondered the possibilities of flight.

As a side note, it is always interesting to gauge the reactions of the chosen participant. Most adults will simply hold the thing and say they have no idea how to make it fly. Children, independent of age, might express doubt as to their "flying" ability, but will inevitably give it a good try. The adults have to be heavily coerced to take the chance!

If the 'copter flew at all, up or down, we ask the volunteer to explain why it flew. The usual answer is that the wings are shaped to make the air move differently around them. The volunteer is then congratulated on this succinct explanation of Bernoulli's Principle. The instructor demonstrates the copter flying up and down, explaining how the direction of the rotation changes the flight. Using a second volunteer with his/her arms held straight out from the body mimicking a jet's wings, we give a very simple explanation of Bernoulli's Principle, explaining how the speed of the airflow around the "wings" creates different air pressure above and below, thus creating lift. If time permits, the paper can be used to have a team paper airplane-building contest and flight "test" to further demonstrate the properties of flight. 


\section{Chemical Engineering}

Again, the descriptive name hints to the audience what kind of materials this engineer might work with. Simple equipment suffices: a glass bottle with snug fitting cork, vinegar, baking soda and a paper towel, and safety glasses. The audience volunteer is a chemical engineer called in to test a new chemical reaction to determine commercial feasibility. For this demonstration, we use a tall glass bottle with a well fitting cork, filled about $1 / 4$ full with white vinegar (exact measurements are not important here). The volunteer, after donning safety glasses, puts baking soda in a small square of paper towel, wraps it up, pushes it into the bottle and puts the cork in. It is refreshing to note that no matter what the age or professed familiarity of the audience with this common reaction, it never fails to garner enthusiasm! The popping of the cork is always a surprise, and inevitably leads to calls of "do it again!"

This precipitates a discussion about the usefulness of controlled explosions. We take this opportunity to tell the children about how the space shuttle, familiar to all, is launched using this principle of a controlled explosion. They are familiar with the orange rocket boosters attached to the shuttle assembly at launch; what they don't always know is that these are filled with liquid oxygen that is ignited to create a controlled explosion of a great enough magnitude to propel the spacecraft out of the Earth's gravity. We remind them that if they have watched a shuttle launch for several minutes, they have seen the rocket boosters drop off to fall into the ocean, where the Navy or Coast Guard, using SONAR, retrieves them.

\section{Civil Engineering}

This demonstration is a team effort. A group of volunteers is given special materials (toothpicks, gumdrops, and a small plastic container) and directed to build a bridge that will span the container. While the group is working, we explain civil engineers as those who specialize in the design and construction of things: buildings, roads, bridges, etc. We discuss the breadth of this profession, and also the myriad factors that go into structural design, including safety, wind, ground conditions, unique weather factors, etc. At the end, we ask the bridge building group to demonstrate their finished product. Elements of design strength are demonstrated here, using gumdrop-and-toothpick squares and triangles.

\section{Electrical Engineering}

For this demonstration, we use a Tesla coil, fluorescent light tubes, the circuit board from a discarded electrical appliance and occasionally a Van de Graaf generator and an incandescent light bulb, hammer, and thick towel. Electrical engineers, we explain, work in a wide variety of

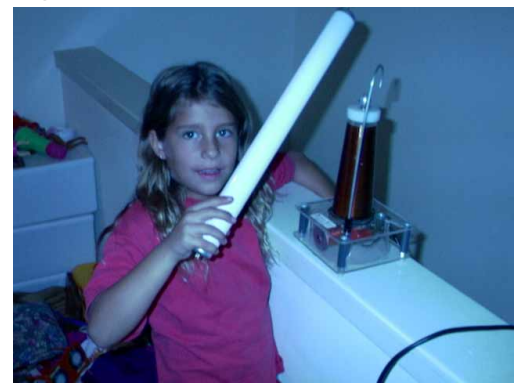
areas of expertise: telephony, circuits and power being but a few.

Proceedings of the 2001 American Society for Engineering Education Annual Conference \& Exposition, Copyright 2001, American Society for Engineering Education 
children, this is a great machine to use to demystify the concept of lightning.) Next, we ask Volunteer \#2 to show us the electromagnetic field the coil is creating. Most will look at us blankly, literally having no idea what to do. As we encourage them, we gently move them closer to the coil, which will, of course, cause the tube to glow. We take this opportunity to explain that in the presence of an electromagnetic field, the nitrogen gas in the fluorescent tube glows. Next, we discuss the principle of electromagnetic fields, and query the audience about their usage of them that day. The point here, of course, is to remove the "awe" aspect of electricity by placing it firmly in their everyday world.

This discussion can be expanded to include incandescent bulbs and how they work. To do this, wrap the bulb in the towel and gently hit it with the hammer. The force of the hit is key; hitting too hard will cause the tungsten wire inside to break as well. Having a grown up deliberately break a light bulb, combined with the ability to see inside it, is a real hit with children. From current electricity, the presenter can move to static electricity, using balloons and the Van de Graaf generator to demonstrate the concept. Finally, a circuit board from an electrical appliance or a computer is used to discuss circuits and their components.

\section{Industrial Engineering}

This is not a widely understood area, and so presents a good opportunity for information. About 20 sheets of paper and a watch or stopwatch are needed for this demonstration. Industrial engineers, we explain, are experts in processes. Manufacturing and the efficient use and maximization of resources are common areas of focus for these professionals. To demonstrate the concept, several volunteers are sought from the audience to form a manufacturing team. The session leader acts as the customer, and requests a bid from the "manufacturing" company for the precise folding of a million pieces of paper each day. The team leader works with the team to determine the folding process, including measuring how long it takes by having the team fold a number of pieces of paper and dividing the total time by the number of papers.

At this point, the instructor can lead the discussion through the correlation between process time, resource requirements and eventually cost and price, explaining the vital role of the industrial engineer throughout. Quality control is also mentioned, and an additional volunteer can be brought up to inspect the quality of each piece of work. This demonstration is best suited to students in grade five and higher, as division is necessary to calculate how long it takes to fold one piece of paper.

\section{Mechanical Engineering}

This is one of the broadest, and therefore more difficult to define, areas of expertise. We explain mechanical engineering as a type of engineering that deals with moving parts. This simple statement covers the wide range of engineering skill areas mechanical engineers work in. The tools used to demonstrate are toys, specifically a Hoberman Sphere and a catapult (using marshmallows as the ammunition).

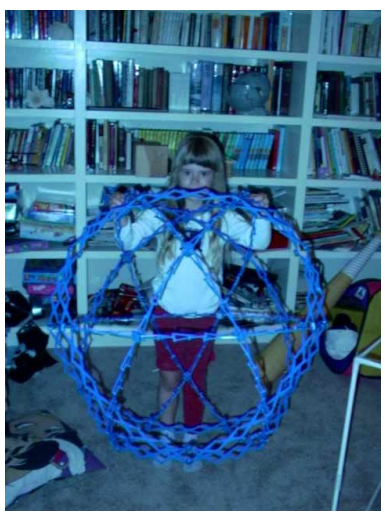

Proceedings of the 2001 American Society for Engineering Education Annual Conference \& Exposition, Copyright 2001, American Society for Engineering Education 
The Hoberman Sphere is an expanding geodesic design that fascinates children and adults alike. It is perfect for explaining the marriage of moving parts and mathematics mechanical engineers work with. The sphere is constructed of parts that fit together in a series of equal triangles, pentagons and stars-a wonderful way to demonstrate how math can be used to design fun things! The leader uses the sphere in various ways to show how safety and repeatability are especially important in toy design.

Next, catapults are presented as mathematical solutions to the problem of enemy attack! Two teams of audience volunteers (teachers vs. students or boys vs. girls work well) are given a catapult and several marshmallows and instructed to fire upon the opposing team on the opposite side of the room. Through trial and error, each group finds the angle required to send the marshmallow flying! The leader uses the battle time to explain that using math (and knowing certain information), the marshmallow can be repeatedly sent exactly where desired. The catapults used are gleaned from the previous year's freshman engineering design day competitions.

\section{Textile Engineering}

This type of engineering is demonstrated using a crowd favorite, fire. Textile engineers, the session leader explains, work with fiber and materials to extend material life or usability or to elicit other desirable qualities from fibers. The equipment consists of three fiber samples (regular cotton, cotton treated with flame retardant, and gun cotton), a candle and holder, matches, and tongs.

The audience is first shown how the regular cotton, the basis of many of today's fashions, bursts into flames when held over a lit candle. Most house fires start at night when the occupants are sleeping, so lives could be saved if sleepwear could be made from a material that would not burn. Next, the leader holds the flame retardant cotton over the flame, demonstrating that it will turn black, but will not flame up. Sometimes, the leader then explains, it is desirable to make a material explode into flames quickly, such as in fireworks for ignition. The gun cotton is then held over the flame, and its quick burst of fire is startling (and VERY fast!). Kevlar thread is also used to demonstrate its strength versus cotton thread.

\section{Materials Engineering}

Materials engineering may be the least commonly known field of engineering. But, of course, the leader explains, everything has to be made out of something. Since materials engineers could work with any other type of engineer, the leader can list examples of electronic materials, specialized ceramics, ammunitions experts, space materials, composites (like mud bricks with and without straw), etc. perhaps passing around examples. As a demonstration, a volunteer is asked to crumple a sample of nitinol wire, and the leader then passes it through the flame of a candle or a match. (Hot water will suffice to straighten the wire, but the candle is already available from the textiles demonstration.) If the audience is large, the wire can be held over the glass of an overhead projector before and after crumpling and heating.

Proceedings of the 2001 American Society for Engineering Education Annual Conference \& Exposition, Copyright 2001, American Society for Engineering Education 


\section{Conclusion}

The conclusion of the program usually revolves around taking the cover off of an old CD player and asking the audience to consider which types of engineers may have worked on this device. Most get electrical and mechanical, but materials engineers could have worked on various aspects, including the material the CD itself is made from. Industrial engineers could have designed the front panel and the layout and manufacturing protocol, etc. The final thought for the program is that most of today's applications are the work of several different types of engineers working as a team.

Assessment of the impact of this presentation is ongoing. To date, only anecdotal evidence is available, but every presentation has resulted in at least one comment that a student is considering engineering who may not have done before. Frequently, these comments refer to women and under-represented minorities. Every presentation has received overwhelmingly positive feedback.

Engineering is becoming an increasingly popular field of study for today's college student. However, the explanation of the practical aspect of what an engineer does is still difficult for most, even experienced, professionals. The session described in this paper is a low-key, relatively non-technical approach to the problem of illuminating the field. The intent is to inform the audience about the fundamental aspects of becoming an engineer; primarily, that engineers use math and science to solve problems with practical solutions.

The demonstrations are intended to further elucidate the many fields of specialization within the profession, while balancing the need to maintain the interest and enthusiasm of the non-technical audience. Examples are not intended to show a high tech, gee whiz approach to engineering. Rather, it is the intent of the program to present engineering as a non-threatening and achievable career path for all, by introducing the concepts of the profession to young children, and explaining the practice to older students and adults. Adult audience members have commented numerous times that they finally understand what engineers do after participating in an "Illuminating Engineering" program!

LAURA J. BOTTOMLEY is the Director of the Women in Engineering and Outreach Programs at North Carolina State University and co-owner of Science Surround, a science education business for children. Dr. Bottomley received her Ph.D. in electrical engineering from North Carolina State University in 1992, and her MSEE and BSEE from Virginia Tech in 1984 and 1985, respectively. She has worked at AT\&T Bell Labs and Duke University.

ELIZABETH A. PARRY is a former manager at IBM. She obtained her BS degree in engineering management with a minor in mechanical engineering from the University of Missouri-Rolla in 1983. She worked for a number of years at IBM before resigning to be a mom. She is now a co-owner of Science Surround and consults for the College of Engineering at North Carolina State University.

Proceedings of the 2001 American Society for Engineering Education Annual Conference \& Exposition, Copyright 2001, American Society for Engineering Education 